\title{
Spin fluctuations and unconventional pairing in $\mathrm{KFe}_{2} \mathrm{As}_{2}$
}

\author{
Katsuhiro Suzuki ${ }^{1,3}$, Hidetomo Usui ${ }^{2}$, and Kazuhiko Kuroki ${ }^{1,3}$ \\ ${ }^{1}$ Department of Engineering Science, The University of Electro-Communications, Chofu, Tokyo 182-8585, Japan \\ ${ }^{2}$ Department of Applied Physics and Chemistry, The University of Electro-Communications, Chofu, Tokyo 182-8585, Japan and \\ ${ }^{3}$ JST, TRIP, Chofu, Tokyo 182-8585, Japan
}

(Dated: August 24, 2021)

\begin{abstract}
We study the relation between the spin fluctuation and superconductivity in a heavily hole doped end material $\mathrm{KFe}_{2} \mathrm{As}_{2}$. We construct a five orbital model by approximately unfolding the Brillouin zone of the three-dimensional ten-orbital model obtained from first-principles calculation. By applying the random-phase-approximation, we obtain the spin susceptibility and solve the linearized Eliashberg equation. The incommensurate spin fluctuation observed experimentally is understood as originating from interband interactions, where the multiorbital nature of the band structure results in an electron-hole asymmetry of the incommensurability in the whole iron-based superconductor family. As for superconductivity, $s$-wave and $d$-wave pairings are found to be in close competition, where the sign change in the gap function in the former is driven by the incommensurate spin fluctuations. We raise several possible explanations for the nodes in the superconducting gap of $\mathrm{KFe}_{2} \mathrm{As}_{2}$ observed experimentally.
\end{abstract}

\section{INTRODUCTION}

A number of interesting features of the iron-based superconductors have attracted much attention. Among them is the material dependence of the superconductivity. Not only the transition temperature $T_{c}$, but also the form of the superconducting gap seems to be material dependent. For instance, although a number of experiments suggest that the gap is fully open on the Fermi surface on some of the arsenides, there are several experiments suggesting the presence of nodes in the superconducting gap $\mathrm{LaFePO}^{1} \underline{\underline{-3}}, \mathrm{BaFe}_{2}\left(\mathrm{As}_{1-x} \mathrm{P}_{x}\right)_{2}{ }^{\underline{4}} \underline{\underline{6}}$, or in $\mathrm{LiFeP}^{7}$. Also in the electron-doped 122 systems, $\mathrm{Ba}\left(\mathrm{Fe}_{1-x} \mathrm{M}_{x}\right)_{2} \mathrm{As}_{2}(\mathrm{M}=\mathrm{Co}, \mathrm{Ni})$, the presence of gap nodes has been suggested 8.9

For LaFePO, theoretical studies suggest the presence of nodes on the electron Fermi surface around the wave vector $(\pi, 0) /(0, \pi)$ in the unfolded Brillouin zone $\underline{\underline{10}-14}$ From this viewpoint, the heavily hole doped end material $\mathrm{KFe}_{2} \mathrm{As}_{2}$ is of special interest because in this material, the electron pockets present in most of the ironbased superconductors are known to be absent due to the low position of the Fermi energy $\stackrel{15,16}{16}$ Nonetheless, the material becomes superconducting, and interestingly, several experiments suggest the presence of nodes in the gap $\stackrel{17-20}{-2}$ Since there are no appreciable electron pockets in $\mathrm{KFe}_{2} \mathrm{As}_{2}$, the nodes are likely to be on the hole Fermi surface. Theoretically, a possibility of $d$-wave pairing has been proposed recently 21,22

Another interesting observation in $\mathrm{KFe}_{2} \mathrm{As}_{2}$ is the spin fluctuations found in neutron-scattering experiments. In Ref. 23, incommensurate spin fluctuation has been observed, and we have theoretically provided its explanation in terms of the multiorbital nature of the Fermi surface.

Given this background, the aim of the present study is to understand the relation between the spin fluctuations and the superconducting gap of $\mathrm{KFe}_{2} \mathrm{As}_{2}$. We first construct a ten-orbital model for $\mathrm{KFe}_{2} \mathrm{As}_{2}$ and also ob- tain a five-orbital model by approximately unfolding the Brillouin zone. We adopt three-dimensional models in order to take into account the possible variation of the superconducting gap along the $k_{z}$ direction. We apply random phase approximation to these models and compare the calculation results at high temperature, which gives reasonable agreement, although the Brillouin zone unfolding is not exact. Then, we go down to low temperature using the five-orbital model. We first summarize our understanding on the origin of the incommensurate spin fluctuations ${ }^{23}$ and also analyze its strength when the material composition is varied from $\mathrm{BaFe}_{2} \mathrm{As}_{2}$ to $\mathrm{KFe}_{2} \mathrm{As}_{2}$. We finally discuss the superconducting gap when Cooper pairing is mediated by spin fluctuations. An analysis based on the linearized Eliashberg equation shows that $s$-wave and $d$-wave pairings are found to be in close competition, and the former is found to have essentially the $s \pm$-wave form in the sense that the gap function around the wave vector $(0,0)$ (where the hole Fermi surfaces exist) and around $(\pi, 0) /(0, \pi)$ (where the electron Fermi surface would have existed in a less doped material) has the opposite sign. This sign change is driven by the incommensurate spin fluctuation, which is due to the interband interaction between states around $(0,0)$ and $(\pi, 0) /(0, \pi)$. Since the electron Fermi pockets are absent, the latter states are somewhat away from the Fermi level, so that this interband sign change of the gap function should not be detected experimentally. As for the possible explanation of the nodes of the gap observed experimentally, we raise several possibilities concerning the hole Fermi surface.

\section{BAND STRUCTURE AND FERMI SURFACE}

First we perform a first-principles band calculation for $\mathrm{KFe}_{2} \mathrm{As}_{2}$ adopting the experimentally determined lattice structure 24 and using the QUANTUM ESPRESSO package $\stackrel{25}{2}$ Then we exploit maximally localized Wannier 
orbitals 26 to obtain the ten-orbital model, whose band structure and the Fermi surface are shown in Fig. 1 (a). There are ten $3 d$ bands because there are two iron atoms per unit cell in the body-centered tetragonal (BCT) lattice structure. Around the $\mathrm{X}$ point, the electron Fermi (a)
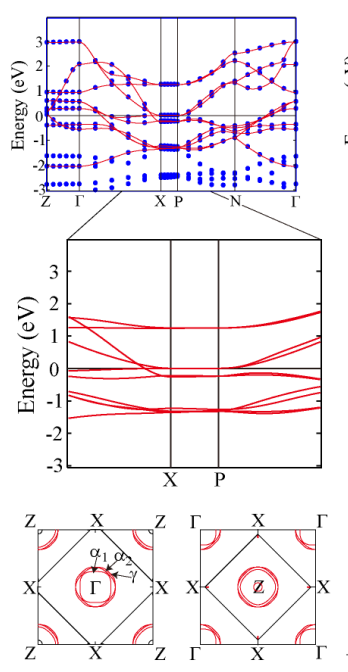

(c)

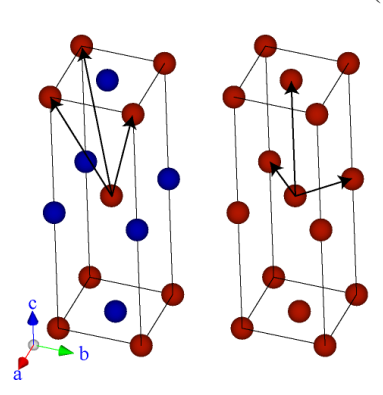

(b)
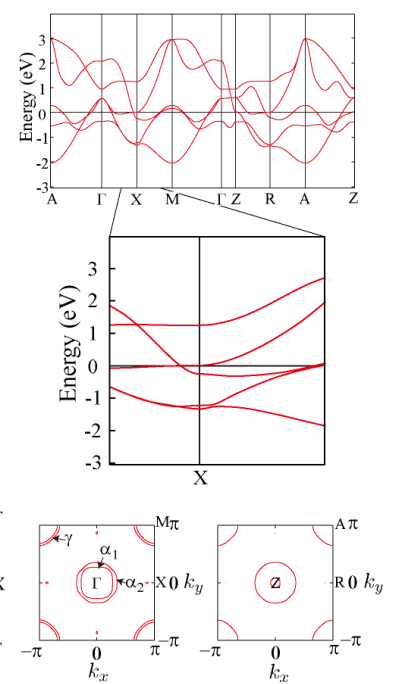

(d)

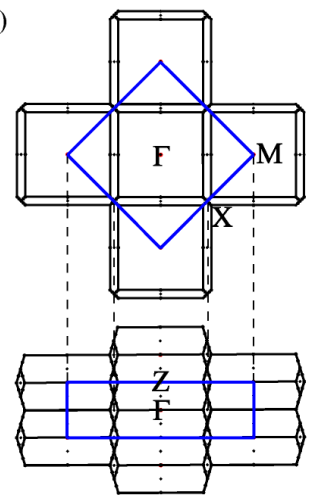

FIG. 1: (color online) Band structure and Fermi surfaces of (a) the ten-orbital model in the original Brillouin zone of the BCT lattice structure and (b) the five-orbital model of $\mathrm{KFe}_{2} \mathrm{As}_{2}$ presented in the unfolded Brillouin zone of the ST lattice structure. The dots in the left are the original firstprinciples band calculation, while the solid lines are the tightbinding model band dispersion obtained by using maximally localized Wannier orbitals. The horizontal cuts of the Fermi surface at $k_{z}=0$ and $k_{z}=\pi$ are presented in the bottom panels of (a) and (b). Translating the left (right) panel of the five-orbital Fermi surface (b) by $(\pi, \pi, \pi)$ and superposing it to the right (left) corresponds to the ten-orbital Fermi surface shown in the right (left). (c) The BCT and the ST lattice structure. The former (latter) unit cell with two (one) iron(s) is adopted for the ten (five)-orbital model. The figure has been produced using VESTA ${ }^{27}$. (d) The Brillouin zone correspondence between the ten-orbital (black line) and fiveorbital (blue line) models. The $\mathrm{Z}$ point of the second Brillouin zone of the BCT structure coincides with the M point of the unfolded Brillouin zone. surfaces, which are present in iron pnictides with fewer holes, are absent. Nonetheless, the band that gives rise to the electron Fermi surface for smaller number of holes sits very close to the Fermi level. Three cylindrical hole Fermi surfaces are present around the $\Gamma$ point, which we name $\alpha_{1}, \gamma$, and $\alpha_{2}$ from the inner one to the outer. Also around the $\mathrm{Z}$ point there is a tiny hole Fermi pocket.

For 122 systems, the Brillouin zone unfolding procedure $\frac{28}{2}$ that adopts the reduced unit cell (with one iron) cannot be done strictly,$\underline{8}$ namely, the hopping integrals that go out from and come into Fe1 and Fe2 in Fig. 11(c) are somewhat different. Nonetheless, if we adopt a reduced unit cell by neglecting the difference, we end up with an approximate five-orbital model. ${ }^{29}$ We show the band structure and the Fermi surface of this model in Fig. 1 (b) in the "unfolded" Brillouin zone of the simple tetragonal (ST) lattice structure. The correspondence between the unfolded ST and the folded BCT Brillouin zone is shown in Fig. 1(d). As seen from this correspondence, the band structure of the five-orbital model can be refolded into the original $\mathrm{BCT}$ Brillouin zone by
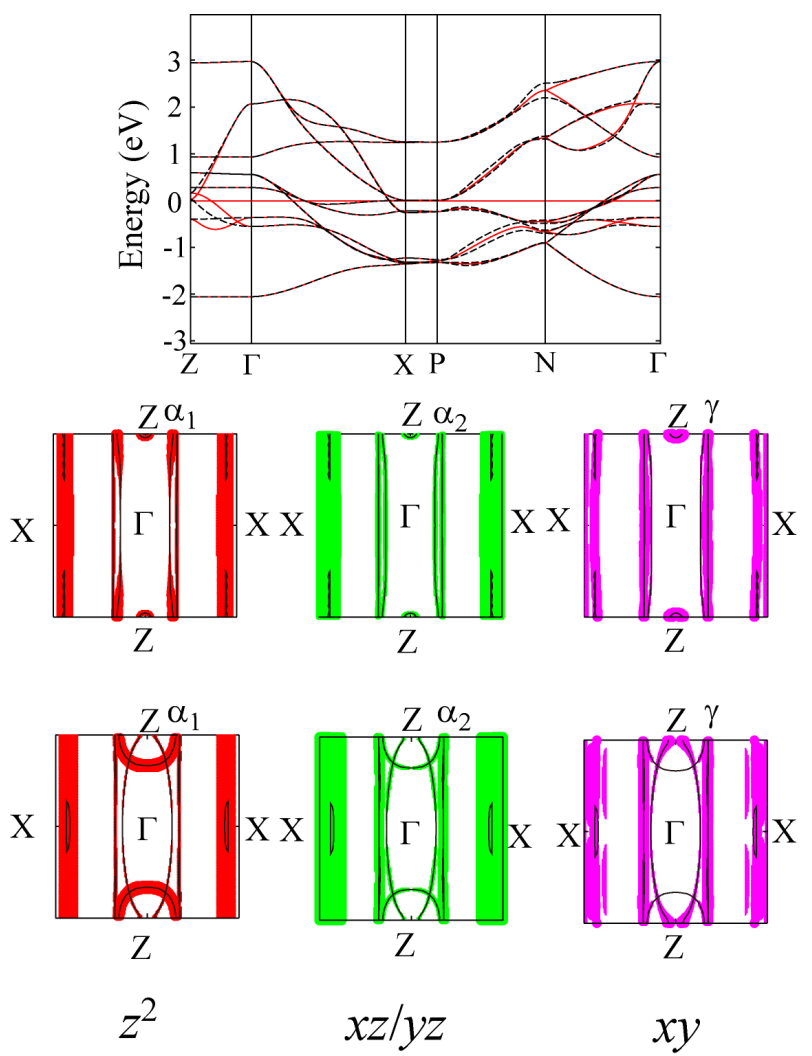

FIG. 2: (Color online) (Top) The band structure of the fiveorbital model of $\mathrm{KFe}_{2} \mathrm{As}_{2}$ refolded into the original Brillouin zone (solid red), and the band structure of the ten-orbital model (dashed black). (Bottom panels) Horizontal cuts of the Fermi surface for the ten-orbital (upper) and five-orbital (lower) models. The thickness represents the $z^{2}, x z / y z$ and $x y$ orbital characters. 
translating the bands in the second BCT Brillouin zone by a wave vector $(\pi, \pi, \pi)$. The comparison of the band structure between the original ten-orbital model and the five-orbital model in the refolded Brillouin zone is shown in Fig. 2. They coincide with each other in most of the portions, but there is some discrepancy along $\Gamma-Z$.

In the middle panels of Fig. 2, we show the vertical cuts of the Fermi surface, where the thickness represents the strength of the $z^{2}, x z / y z$, or $x y=\left(X^{2}-Y^{2}\right)$ orbital character. All of the hole Fermi surfaces are very cylindrical, so that the two-dimensionality is strong. However, if we look at the orbital character, $\alpha_{2}$ and $\gamma$ Fermi surfaces have strong $x z / y z$ and $x y$ character, respectively, regardless of $k_{z}$, while the $\alpha_{1}$ Fermi surface is mainly composed of $z^{2}$ orbital around the $\mathrm{Z}$ point, while the $x z / y z$ character becomes stronger around the $\Gamma$ point. In this sense, $\alpha_{1}$ can be considered as less two-dimensional compared to the other two-hole Fermi surfaces.

In the bottom panels of Fig. 2, we show similar vertical cuts of the Fermi surface for the five-orbital model, refolded into the original Brillouin zone. The result in most portions is close to that obtained for the ten-orbital model, but the $\alpha_{1}$ hole Fermi surface, which is a cylinder in the latter, splits into a three-dimensional pocket around the $\mathrm{Z}$ point and a strongly warped cylinder. This split occurs between portions of the Fermi surface having strong $z^{2}$ and $x z / y z$ character. Although this may seem like a large difference, the effect of this splitting is not so large as far as the quantities we focus on are concerned, as we shall see later.

\section{MANY-BODY HAMILTONIAN AND RANDOM-PHASE APPROXIMATION}

For the many-body part of the Hamiltonian, we consider the intraorbital $U$, the interorbital $U^{\prime}$, the Hund's coupling $J$, and the pair hopping interaction $J^{\prime}$. We consider orbital-dependent interactions,, 30 and the interaction part of the Hamiltonian is given as

$$
\begin{aligned}
\mathcal{H}^{\prime}= & \sum_{i}\left(\sum_{\mu} U_{\mu} n_{i \mu \uparrow} n_{i \mu \downarrow}+\sum_{\mu>\nu} \sum_{\sigma, \sigma^{\prime}} U_{\mu \nu}^{\prime} n_{i \mu \sigma} n_{i \mu \sigma^{\prime}}\right. \\
& \left.-\sum_{\mu \neq \nu} J_{\mu \nu} S_{i \mu} \cdot S_{i \nu}+\sum_{\mu \neq \nu} J_{\mu \nu}^{\prime} c_{i \mu \uparrow}^{\dagger} c_{i \mu \downarrow}^{\dagger} c_{i \nu \uparrow} c_{i \nu \downarrow}\right),
\end{aligned}
$$

where $i$ denotes the sites and $\mu, \nu$ stand for the orbitals. We apply random-phase approximation (RPA) to this model and obtain the spin and charge susceptibility matrices. Namely, using the bare Green's function $G_{l m}(k)\left(k=\left(\boldsymbol{k}, i \omega_{n}\right)\right)$ in the orbital representation, the irreducible susceptibility matrix is given as

$$
\chi_{l_{1}, l_{2}, l_{3}, l_{4}}^{0}(q)=\sum_{k} G_{l_{1} l_{3}}(k+q) G_{l_{4} l_{2}}(k)
$$

The spin and the charge (orbital) susceptibility matrices are obtained as

$$
\begin{gathered}
\chi_{s}(q)=\frac{\chi^{0}(q)}{1-S \chi^{0}(q)} \\
\chi_{c}(q)=\frac{\chi^{0}(q)}{1+C \chi^{0}(q)}
\end{gathered}
$$

where $S$ and $C$ are interaction vertex matrices. We refer to the largest eigenvalue of the matrices $S \chi^{0}(q)$ and $\chi_{s}(q)$ for $i \omega_{n}=0$ as the Stoner factor and the spin susceptibility, respectively. The magnetic instability is signaled by the Stoner factor exceeding unity. In the RPA calculation (where the self-energy correction is neglected), adopting the interaction values evaluated by first-principles calculation ${ }^{30}$ easily results in a magnetic instability even at high temperature, so we multiply all the electron-electron interaction by a constant factor $f \underline{10}$.

To analyze superconductivity, the Green's function and the effective singlet pairing interaction,

$$
V^{s}(q)=\frac{3}{2} S \chi^{s}(q) S-\frac{1}{2} C \chi^{c}(q) C+\frac{1}{2}(S+C),
$$

are plugged into the linearized Eliashberg equation,

$$
\begin{aligned}
\lambda \phi_{l_{1} l_{4}}(k)= & -\frac{T}{N} \sum_{q} \sum_{l_{2} l_{3} l_{5} l_{6}} V_{l_{1} l_{2} l_{3} l_{4}}(q) G_{l_{2} l_{5}}(k-q) \\
& \times \phi_{l_{5} l_{6}}(k-q) G_{l_{3} l_{6}}(q-k)
\end{aligned}
$$

The eigenvalue $\lambda$ increases upon lowering the temperature and reaches unity at $T_{c}$. Instead of going down to $T_{c}$, which will be a tedious calculation, we perform the calculation at a fixed temperature and obtain the eigenvalue and the eigenvector for $s$-wave and $d$-wave pairing symmetries. The comparison of the eigenvalue at a fixed (low) temperature for different pairing symmetries tells us which pairing state is actually realized in the system. The eigenfunction of the Eliashberg equation is referred to as the superconducting gap function.

\section{COMPARISON BETWEEN TEN- AND FIVE-ORBITAL MODELS}

We first compare the five- and ten-orbital models at a high temperature of $T=0.07 \mathrm{eV}$. We take $16 \times 16 \times 16$ $k$-point meshes and 128 Matsubara frequencies. The interaction reducing factor is $f=0.53$. The Stoner factor calculated for this parameter set is $\alpha_{S}=0.94$ and 0.95 for the ten- and five-orbital models, respectively, in reasonable agreement. In Fig. 3, we show the spin susceptibility along the wave vector $(0,0)$ to $(\pi, 0)$ in the unfolded Brillouin zone. The slight difference in the Stoner factor results in a difference in the peak value of the spin susceptibility, but the $q_{x}$ position at which the spin susceptibility is maximized is the same between the two models. It can 


\section{0 orbital model}
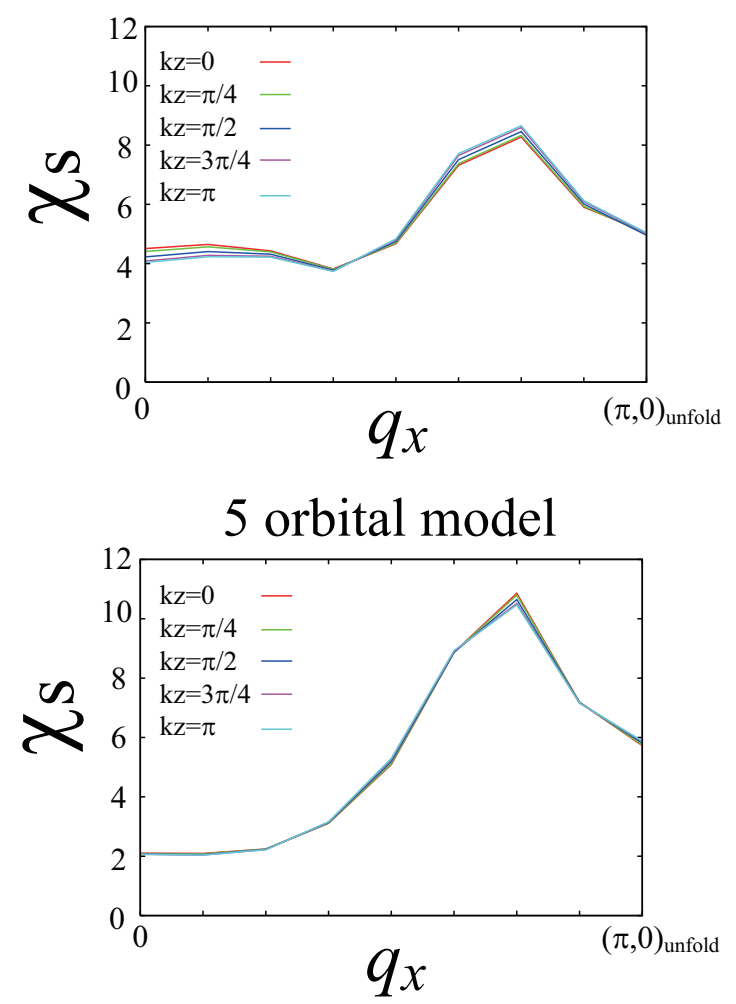

FIG. 3: (Color online) Comparison of the spin susceptibility between the ten-orbital (top) and five-orbital (bottom) models along the wave vector $(0,0)$ to $(\pi, 0) . T=0.07 \mathrm{eV}$ with $16 \times 16 \times 16 k$-point meshes.

also be seen from this figure that the three-dimensionality of the spin fluctuations is very small.

In Fig 4, we compare the superconducting gap for the two models in the folded Brillouin zone. For the fiveorbital model, the results are obtained first in the unfolded Brillouin zone, and then those in the second Brillouin zone of the original BCT lattice structure is moved to the first Brillouin zone. Here again they have similar structures for most of the $k_{z}$ planes given here. The eigenvalue of the Eliashberg equation is 0.66 and 0.76 for $s$-wave, and 0.51 and 0.60 for $d$-wave for the ten- and five-orbital models, respectively. Since the Fermi surface is different between the two models for some $k_{z}$ portions, the gap function differs between the two at those portions of the Fermi surface. Nonetheless, the present comparison shows that the five-orbital model can be considered as reliable as far as the form of the superconducting gap on most of the $k_{z}$ planes are concerned. Hereafter, we concentrate on the five-orbital model and go down to lower temperatures, where larger-number $k$-point meshes and Matsubara frequencies are required. (a) 10 orbital model hole hole hole

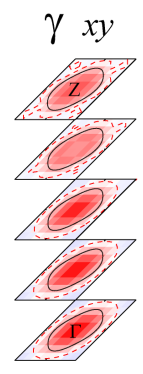

$\alpha_{1} \quad z^{2} / x z / y z$

$\alpha_{2} x z / y z$

electron
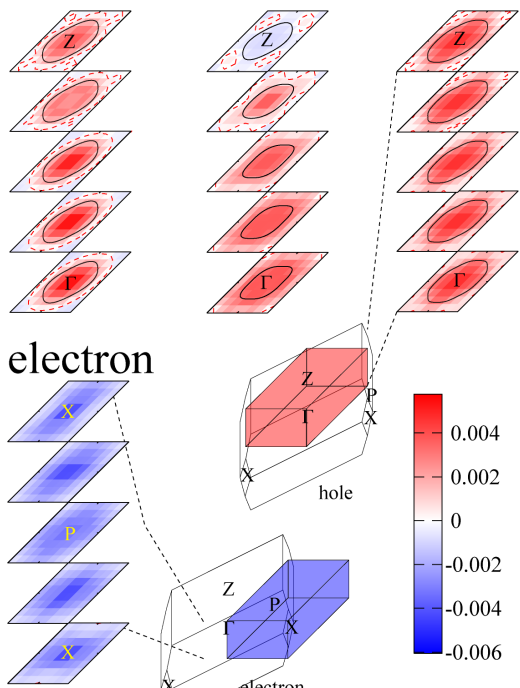

(b) 5 orbital model

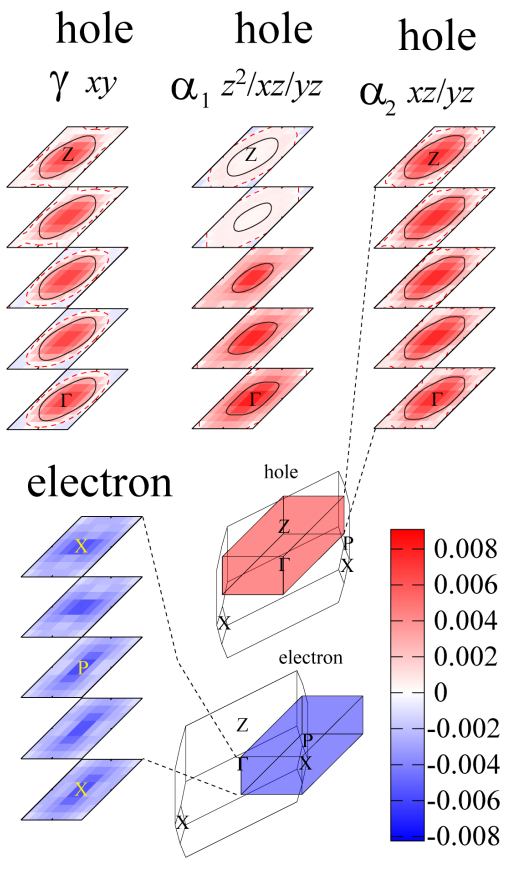

FIG. 4: (Color online) Comparison of the $s \pm$ gap function between (a) the ten-orbital and (b) the five-orbital models at $T=0.07 \mathrm{eV}$ with $16 \times 16 \times 16 k$-point meshes. Contour plots are shown for five horizontal cuts around $\Gamma-\mathrm{Z}$ and $\mathrm{X}-\mathrm{P}-\mathrm{X}$ in the original Brillouin zone of the BCT lattice. The five-orbital results, obtained originally in the unfolded Brillouin zone, are refolded into this Brillouin zone. Solid black lines are the Fermi surface and the red dashed lines are the nodal lines of the gap. The Brillouin zone is shown along with the regions where the gap is presented. 
(a) $n=5.5$

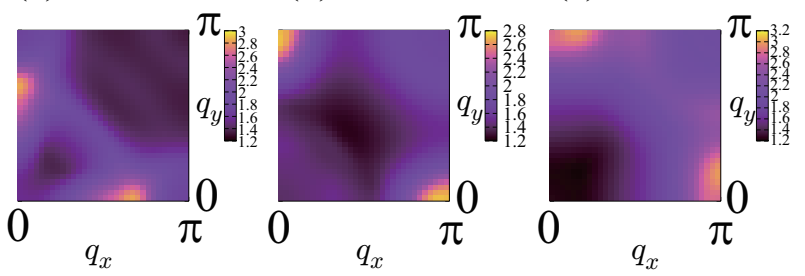

(d)

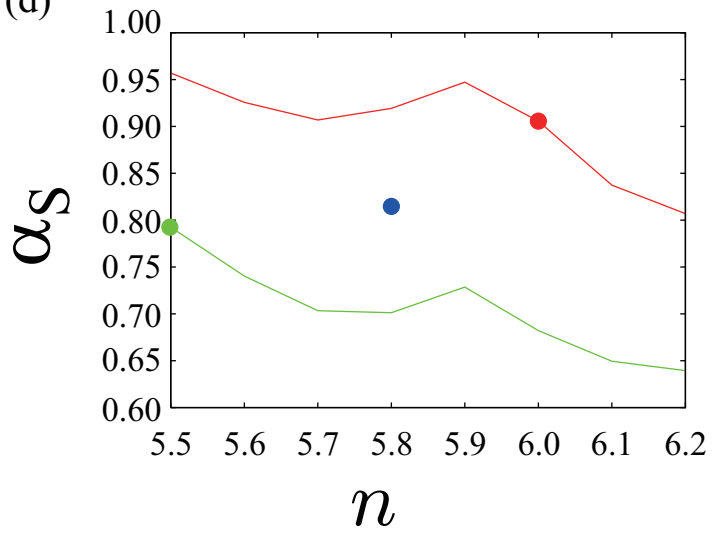

FIG. 5: (Color online) The spin susceptibility for the five-orbital models at $T=0.04 \mathrm{eV}$ with (a) $n=5.5$ $\left(\mathrm{KFe}_{2} \mathrm{As}_{2}\right)$, (b) $n=5.8\left(\mathrm{Ba}_{0.6} \mathrm{~K}_{0.4} \mathrm{Fe}_{2} \mathrm{As}_{2}\right)$, and (c) $n=6.1$ $\left(\mathrm{Ba}\left(\mathrm{Fe}_{0.9} \mathrm{Co}_{0.1}\right)_{2} \mathrm{As}_{2}\right)$. (d) The Stoner factor against the band filling for the models of $\mathrm{KFe}_{2} \mathrm{As}_{2}$ and $\mathrm{BaFe}_{2} \mathrm{As}_{2}$. The dots indicate the positions of the actual band filling of the corresponding material. At $n=5.8$, we adopt the model of $\mathrm{Ba}_{0.6} \mathrm{~K}_{0.4} \mathrm{Fe}_{2} \mathrm{As}_{2}$.

\section{FIVE-ORBITAL MODEL AT LOWER TEMPERATURE}

\section{A. Spin fluctuation}

We now discuss the spin fluctuations using the fiveorbital model. Here we take $T=0.04 \mathrm{eV}$, adopting $64 \times 64 \times 16 k$-point meshes and 512 Matsubara frequencies. One of the merits to adopting the five-orbital model is that it captures the essence of the spin fluctuations observed in neutron-scattering experiments. In Fig 5 , we show the contour plot of the spin susceptibility of the five-orbital model in the unfolded Brillouin zone at $k_{z}=0$. In addition to the results for $\mathrm{KFe}_{2} \mathrm{As}_{2}$ (band filling $n=5.5$ ), we also show for comparison the results for $\mathrm{Ba}_{0.6} \mathrm{~K}_{0.4} \mathrm{Fe}_{2} \mathrm{As}_{2}(n=5.8)$ and $\mathrm{Ba}\left(\mathrm{Fe}_{0.9} \mathrm{Co}_{0.1}\right)_{2} \mathrm{As}_{2}$ $(n=6.1)$. For $\mathrm{Ba}_{0.6} \mathrm{~K}_{0.4} \mathrm{Fe}_{2} \mathrm{As}_{2}$, we use the five-orbital model that is obtained by making a linear combination of the hopping integrals of $\mathrm{BaFe}_{2} \mathrm{As}_{2}$ and $\mathrm{KFe}_{2} \mathrm{As}_{2}$ using the lattice structure of $\mathrm{Ba}_{0.6} \mathrm{~K}_{0.4} \mathrm{Fe}_{2} \mathrm{As}_{2}$ given in Ref. 31, as was done in Ref. 32. For $n=6.1$, we simply use the five-orbital model of $\mathrm{BaFe}_{2} \mathrm{As}_{2}$ adopting the experimental lattice structure $\stackrel{33}{ }$ The interaction reducing
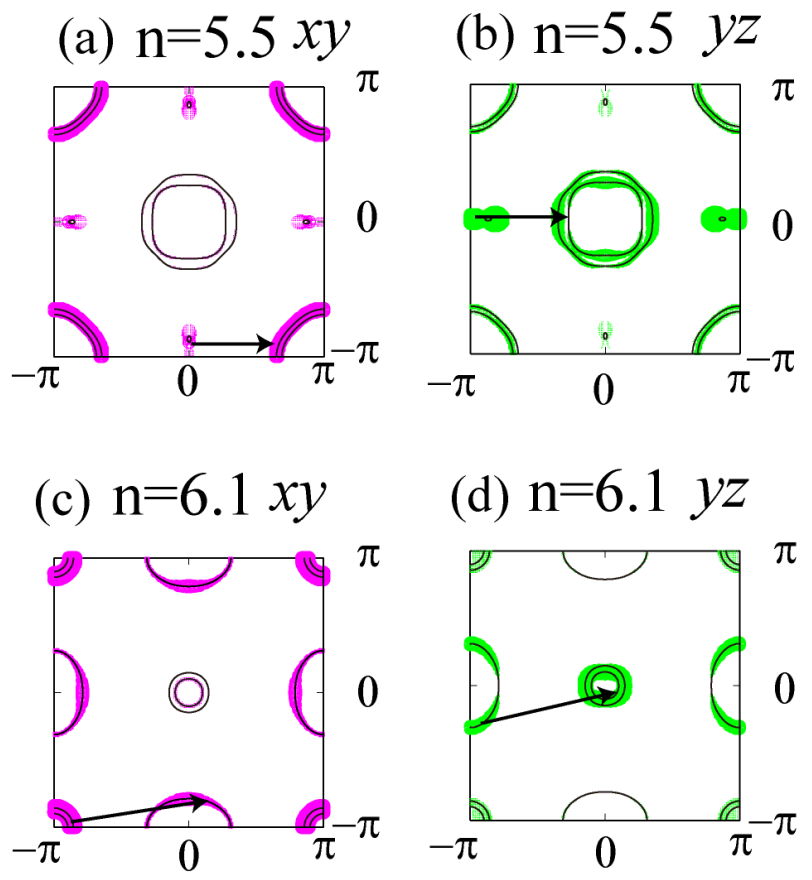

FIG. 6: (Color online) The orbital character distribution on the Fermi surface for $k_{z}=0 . \quad x y(\mathrm{a}),(\mathrm{c})$ and $y z(\mathrm{~b}),(\mathrm{d})$ orbitals for the band fillings $n=5.5$ (a),(b) and $n=6.1$ (c),(d). The arrows indicate the wave vectors connecting the portions of the Fermi surface having similar orbital character. These arrows correspond to the peak position in the spin susceptibility.

rate is taken to be $f=0.40$, so that the Stoner factor does not exceed unity in all of the three cases considered here. In Fig. 5(a), the peak position is located at an incommensurate wave vector $[(1-2 \delta) \pi, 0], \delta=0.17$, which is in very good agreement with a recent neutron-scattering experiment for $\mathrm{KFe}_{2} \mathrm{As}_{2} 23$.

For $\mathrm{Ba}_{0.6} \mathrm{~K}_{0.4} \mathrm{Fe}_{2} \mathrm{As}_{2}(n=5.8)$, the peak moves to the commensurate position $(\pi, 0)$. In the case of electron doping, $n=6.1$, the peak moves toward $(\pi, \pi)$ in the unfolded Brillouin zone, which is consistent with the experimental results of $\mathrm{Ba}(\mathrm{Fe}, \mathrm{Co})_{2} \mathrm{As}_{2}$ in the highenergy regime. The above tendency has also been obtained in other theoretical studies that use realistic band structures,$\frac{12,28,34-37}{2-37}$

In the above, the adopted band structure is different among Figs. 5(a),5(b) and 5(c). To focus on the evolution of the spin fluctuations, we have also fixed the band structure to that of $\mathrm{KFe}_{2} \mathrm{As}_{2}$ or $\mathrm{BaFe}_{2} \mathrm{As}_{2}$, and varied the band filling hypothetically from 5.5 to 6.2 . We find that for both of the band structures, the spin fluctuation becomes commensurate around $n=5.8 \sim 6.0$. For both of the models, it is found that the peak position continuously moves from the commensurate to the incommensurate position as holes are doped, $\underline{\underline{38}}$ indicating that the spin fluctuation has the same origin in the entire 
$(\mathrm{Ba}, \mathrm{K}) \mathrm{Fe}_{2} \mathrm{As}_{2}$ system, namely, the interband scattering between the band around $(0,0)$ and $(\pi, 0) /(0, \pi)$.

The Stoner factor obtained for the above models is shown in Fig. 5(d) as functions of the band filling. This shows that the strength of the spin fluctuations is stronger for the model of $\mathrm{BaFe}_{2} \mathrm{As}_{2}$ than in $\mathrm{KFe}_{2} \mathrm{As}_{2}$ when compared at the same band filling. Therefore, when the composition of the material is changed continuously from Ba to $\mathrm{K}$, we would expect the spin fluctuation to be maximum around the undoped regime (the $\mathrm{Ba}$ rich regime), and decreases when the holes are doped by $\mathrm{Ba} \rightarrow \mathrm{K}$ replacement. However, in the heavily hole doped regime (the K-rich regime), the strength of the spin fluctuations should barely decrease, and may become even stronger with hole doping approaching the end material $\mathrm{KFe}_{2} \mathrm{As}_{2}$.

As for the electron-hole asymmetry of the incommensurability of the spin fluctuations, we have analyzed its origin in Ref. 23 as follows. The spin fluctuations are mainly governed by the interaction between portions of the Fermi surface having similar orbital character, so in Fig. 6(a)-(d), we show the strength of the orbital character on the Fermi surfaces of $\mathrm{KFe}_{2} \mathrm{As}_{2}(n=5.5)$ and $\mathrm{BaFe}_{2} \mathrm{As}_{2}$ with $n=6.1$ in the unfolded Brillouin zone. Here we plot the states within a finite energy range $-\Delta E<E(k)-E_{F}<\Delta E$ with $\Delta E=0.02 \mathrm{eV}$, which can contribute to the spin fluctuations. Although Fermi surfaces near the wave vector $(\pi, 0) /(0, \pi)$ are barely present in $\mathrm{KFe}_{2} \mathrm{As}_{2}$, there are states in the vicinity of the Fermi level originating from a nearly flat band lying close to the Fermi level. The position of this flat band with respect to the Fermi level can be clearly seen in the magnifications of Figs. 1(a) and 1(b). As mentioned in Ref. 10, the spin fluctuations develop at wave vectors which bridge the portions of the Fermi surface having similar orbital character. The wave vectors connecting $x y$ orbital portions and those connecting $y z$ (or $x z$, not shown) portions nearly coincide in each of the two cases, so that this wave vector should correspond to the peak position of the spin fluctuations. In the electron-doped case, the connecting wave vector deviates from $(\pi, 0)$ toward $(\pi, \pi)$, while in the hole-doped case it deviates toward $(0,0)$. Thus, the difference of the spin fluctuation incommensurability between electron- and hole-doped cases originates from the multiorbital nature of the system.

\section{B. Superconducting gap}

Finally, we discuss the superconducting gap of $\mathrm{KFe}_{2} \mathrm{As}_{2}$. We obtain the superconducting gap at $T=$

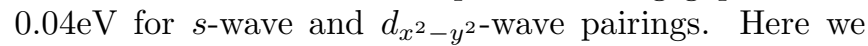
take $32 \times 32 \times 16 k$-point meshes and 1024 Matsubara frequencies. We show in Fig. 7 the $s$-wave (a) and $d$-wave (b) gap functions of the five-orbital model refolded into the original folded Brillouin zone. Here we take the interaction reducing rate $f=0.49$. The rate $f$ is increased compared to the values adopted for the spin suscepti- bility because here we focus only on $\mathrm{KFe}_{2} \mathrm{As}_{2}$, so that the Stoner factor does not exceed unity even for this $f$. We take larger values of the interaction so as to enhance the eigenvalue of the Eliashberg equation, which enables us to obtain a more reliable form of the superconducting gap. The eigenvalue of the Eliashberg equation is $\lambda_{s} \sim 1.01$ and $\lambda_{d_{x^{2}-y^{2}}} \sim 0.98$, close to unity. These values indicate that the pairing symmetry competition is close. In fact, the possibility of $d$-wave pairing was proposed in a recent functional renormalization group study ${ }^{21}$ and an effective Hamiltonian approach ${ }^{22}$ Even at this low temperature, the gap is essentially similar to the one obtained at higher temperature in Sec. IV The $s$-wave gap is basically $s \pm$-wave in the sense that the gap function changes sign between the states near $\boldsymbol{k} \sim(0,0)$ and $\boldsymbol{k} \sim(\pi, 0) /(0, \pi)$. The origin of this sign change is strongly related to the spin fluctuation discussed in the present section. Namely, although the Fermi surface is barely present around $\boldsymbol{k} \sim(\pi, 0) /(0, \pi)$, there are states in the vicinity of the Fermi level originating from the bands that produces the electron Fermi surface for smaller hole content. The pair scattering from those states to the hole Fermi surface and vice versa mediated by the incommensurate spin fluctuations is the origin of the sign change of the gap function. On the other hand, the $d$-wave pairing originates essentially due to the intraband repulsive interaction. In fact, we find that the spin fluctuations originating from the intraband interaction (within the hole Fermi surfaces) develops especially for the band filling below $n=5.5$.

Although the origin of the stabilization of the $s$-wave gap is the incommensurate spin fluctuation and the gap function (eigenfunction of the Eliashberg equation, to be strict) changes its sign between $\boldsymbol{k} \sim(0,0)$ and $\boldsymbol{k} \sim(\pi, 0)$, theoretically, the Fermi surface around the latter wave vector is barely present for $\mathrm{KFe}_{2} \mathrm{As}_{2}$, so, experimentally, this sign change should not be detectable. However, there are several possibilities of (nearly) vanishing gap function, which may be relevant to the experimental observations that suggest the presence of nodes in the superconducting gap. First, the $s$-wave superconducting gap on the $x y$ Fermi surface is rather small. One can see in Fig. 7(a) that the nodal line runs somewhat close to the $x y$ Fermi surface. This may experimentally be detected as nearly vanishing gap, although there is no actual sign change in the gap function here. Second, the $s$-wave gap on the $\alpha_{1}$ Fermi surface has different signs between the $k_{z}$ plane that includes the $\Gamma$ point and that with the $\mathrm{Z}$ point, as seen in Fig. 7(a). This means that the gap has horizontal nodes on the $\alpha_{1}$ Fermi surface, which is schematically shown in Fig. 7(c). Although the five-orbital model is not reliable concerning this portion of the Fermi surface, this sign change is also found in the ten-orbital model (at higher temperature) as seen in Fig. 4 in SecIV The sign change in this $\alpha_{1}$ hole Fermi surface is a common feature found in the RPA studies of the 122 systems, $\stackrel{32,34}{,}$ and indeed some recent experiments $\frac{39,40}{2}$ support this possibility in phosphorous 

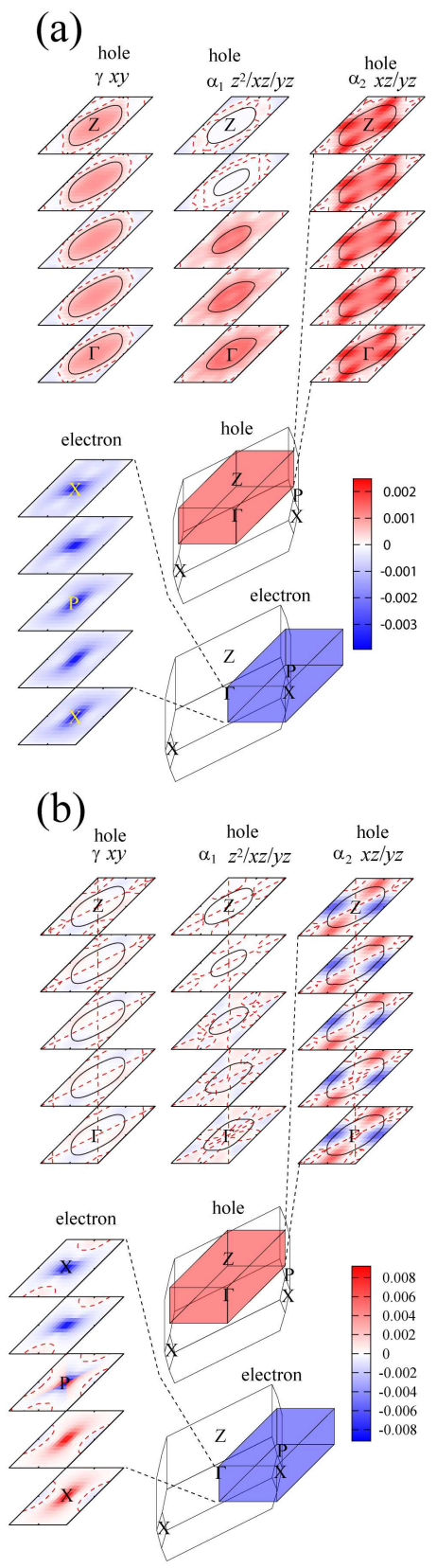

(c)

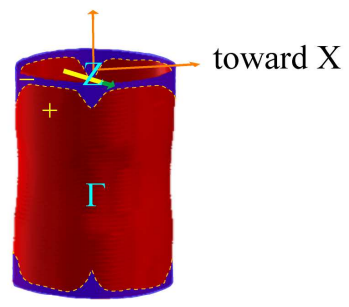

FIG. 7: (Color online) The contour plots of gap function of the five-orbital model for $s$-wave (a) and $d_{x^{2}-y^{2}}$-wave (b) at $T=0.04 \mathrm{eV}$. The solid black lines represent the Fermi surfaces, while dashed red lines are the nodes of the gap. (c) A schematic figure of the horizontal nodes in the $\alpha_{1}$ Fermi surface in the case of $s$-wave pairing. The arrow indicates the intraband scattering that drives the sign change. doped 122 materials. Third, there is a possibility of a $d$-wave gap, which is in close competition with the $s$ wave. This possibility, however, may not be consistent with an experiment on the magnetic vortex lattice, which shows that the gap is not strongly anisotropic within the planes $\underline{\underline{41}}$

\section{CONCLUSIONS}

In the present study, we have constructed an approximate five-orbital model of $\mathrm{KFe}_{2} \mathrm{As}_{2}$ and compared it with the original ten-orbital model. We find the former to be reliable as far as the peak position of the spin fluctuations and form of the gap function are concerned. Using the five-orbital model, we have investigated the spin fluctuations and the spin-fluctuation-mediated superconducting gap of $\mathrm{KFe}_{2} \mathrm{As}_{2}$. We find that the spin fluctuation has an incommensurate peak structure, whose origin is the multiorbital nature of the Fermi surfaces. The peak position of the spin fluctuation is in excellent agreement with the experiment. $\underline{\underline{23}}$

As for the superconductivity, $s$-wave and $d$-wave pairings are found to be in close competition. The $s$-wave gap has essentially the $s$-wave form in the sense that the gap function changes its sign between the wave vectors around $(0,0)$ and $(\pi, 0) /(0, \pi)$. Although there are barely Fermi surfaces around $(\pi, 0) /(0, \pi)$, the states in the vicinity of the Fermi level are nonetheless effective to give the incommensurate spin fluctuations and thus the sign change in the superconducting gap function. Although the sign change exists in the gap function, this should not be detectable in experiments because the Fermi surface is barely present around $(0, \pi) /(\pi, 0)$. As for the explanation for the nodes in the superconducting gap observed experimentally, besides a possible $d$-wave pairing, we raise two possibilities for the $s$-wave pairing: either horizontal nodes in the $x z / y z / z^{2}$ Fermi surface or the small gap on the entire $x y$ Fermi surface.

\section{ACKNOWLEDGMENTS}

We are grateful to C.H. Lee, H. Fukazawa, H. Ikeda, T. Shibauchi Y. Matsuda, H. Eisaki, K. Yamada, T. Shimojima, and K. Okazaki for valuable discussions. Numerical calculations were performed at the facilities of the Information Technology Center, University of Tokyo, and also at the Supercomputer Center, ISSP, University of Tokyo. This study has been partially supported by Grant-in-Aid for Scientific Research from MEXT of Japan and from the Japan Society for the Promotion of Science. 
1 J. D. Fletcher, A. Serafin, L. Malone, J. G. Analytis, J-H Chu, A.S. Erickson, I. R. Fisher, and A. Carrington, Phys. Rev. Lett. 102, 147001 (2009).

2 C. W. Hicks, T. M. Lippman, M. E. Huber, J. G. Analytis, J.-H. Chu, A. S. Erickson, I. R. Fisher, and K. A. Moler, Phys. Rev. Lett. 103, 127003 (2009).

3 M. Yamashita, N. Nakata, Y. Senshu, S. Tonegawa, K. Ikada, K. Hashimoto, H. Sugawara, T. Shibauchi, and Y. Matsuda, Phys. Rev. B 80, 220509(R) (2009).

${ }^{4}$ K. Hashimoto, M. Yamashita, S. Kasahara, Y. Senshu, N. Nakata, S. Tonegawa, K. Ikada, A. Serafin, A. Carrington, T. Terashima, H. Ikeda, T. Shibauchi and Y. Matsuda, Phys. Rev. B 81, 220501(R) (2010).

5 Y. Nakai, T. Iye, S. Kitagawa, K. Ishida, S. Kasahara, T. Shibauchi, Y. Matsuda and T. Terashima: Phys. Rev. B. 81 (2010) 020503(R).

${ }^{6}$ M. Yamashita, Y. Senshu, T. Shibauchi, S. Kasahara, K. Hashimoto, D. Watanabe, H. Ikeda, T. Terashima, I. Vekhter, A.B. Vorontsov, and Y. Matsuda, Phys. Rev. B $84060507(\mathrm{R})$ (2011).

7 K. Hashimoto, S. Kasahara, R. Katsumata, Y. Mizukami, M. Yamashita, H. Ikeda, T. Terashima, A. Carrington, Y. Matsuda, and T. Shibauchi, arXiv:1107.4505.

8 I. I. Mazin , T. P. Devereaux, J. G. Analytis, Jiun-Haw Chu, I. R. Fisher, B. Muschler and R. Hackl, Phys. Rev. B 82, 180502 (2010).

9 C. Martin , H. Kim, R. T. Gordon, N. Ni, V. G.Kogan, S. L. Bud'ko, P. C. Canfield, M. A. Tanatar, R. Prozorov, Phys. Rev. B 81, 060505 (2010).

${ }^{10}$ K. Kuroki, H. Usui, S. Onari, R. Arita and H. Aoki, Phys. Rev. B 79, 224511 (2009).

11 F. Wang, H. Zhai, and D.-H. Lee, Phys. Rev. B 81, 184512 (2010).

12 H. Ikeda, R. Arita, and J. Kuneš, Phys. Rev. B 81, 054502 (2010).

13 T. Kariyado and M. Ogata, J. Phys. Soc. Jpn. 79, 033703 (2010).

14 R. Thomale, C. Platt, W. Hanke, B.A. Bernevig, Phys. Rev. Lett. 106, 187003 (2011).

15 T. Sato, K. Nakayama, Y. Sekiba, P. Richard, Y.-M. Xu, S. Souma, T. Takahashi, G. F. Chen, J. L. Luo, N. L. Wang and H. Ding, Phys. Rev. Lett. 103, 047002 (2009).

16 T. Terashima, M. Kimata, N. Kurita, H. Satsukawa, A. Harada, K. Hazama, M. Imai, A. Sato, K. Kihou, C.-H. Lee, H. Kito, H. Eisaki, A. Iyo, T. Saito, H. Fukazawa, Y. Kohori, H. Harima, and S. Uji, J. Phys. Soc. Jpn. 79, 053702 (2010).

17 H. Fukazawa, Y. Yamada, K. Kondo, T. Saito, Y. Kohori, K. Kuga, Y. Matsumoto, S. Nakatsuji, H.Kito, P.M. Shirage, K. Kihou, N. Takeshita, C. H. Lee, A. Iyo and H. Eisaki, J. Phys. Soc. Jpn. 78, 083712 (2009).

18 J. K. Dong, S. Y. Zhou, T. Y. Guan, H. Zhang, Y. F. Dai, X. Qiu, X. F. Wang, Y. He, X. H. Chen and S. Y. Li, Phys. Rev. Lett. 104, 087005 (2010).

19 S.W. Zhang, L. Ma, Y.D. Hou, J. Zhang, T.-L. Xia, G.F.Chen, J.P. Hu, G.M. Luke and W. Yu, Phys. Rev. B 81, 012503 (2010).

${ }^{20}$ K. Hashimoto , A. Serafin, S. Tonegawa, R. Katsumata, R. Okazaki, T. Saito, H. Fukazawa, Y. Kohori, K. Kihou, C. H. Lee, A. Iyo, H. Eisaki, H. Ikeda, Y. Matsuda, A. Carrington and T. Shibauchi, Phys. Rev. B 82, 014526
(2010).

21 R. Thomale, C. Platt, W. Hanke, J. Hu, and B. A. Bernevig, Phys. Rev. Lett. 107, 117001 (2011).

22 S. Maiti, M. Korshunov, T. Maier, P.J. Hirschfeld, and A.V. Chubukov, Phys. Rev. Lett. 107, 147002 (2011), arXiv:1109.0498

23 C. H. Lee, K. Kihou, H. Kawano-Furukawa, T. Saito, A. Iyo, H. Eisaki, H. Fukazawa, Y. Kohori, K. Suzuki, H. Usui, K. Kuroki and K. Yamada, Phys. Rev. Lett. 106,067003 (2011)

24 S. Rozsa, H. U. Schuster, Z. Naturforsch. B 36, 1668 (1981).

25 S. Baroni , A. Dal Corso, S. de Gironcoli, P. Giannozzi, C. Cavazzoni, G. Ballabio, S. Scandolo, G. Chiarotti, P. Focher, A. Pasquarello, K. Laasonen, A. Trave, R. Car, N. Marzari, A. Kokalj: http://www.pwscf.org/ Here we adopt the exchange correlation functional introduced by J. P. Perdew, K. Burke, and Y. Wang (Phys. Rev. B 54, 16533 (1996)), and the wave functions are expanded by plane waves up to a cutoff energy of $40 \mathrm{Ry} .8^{3} k$-point meshes are used.

26 N. Marzari and D. Vanderbilt, Phys. Rev. B 5612847 (1997); I. Souza, N. Marzari and D. Vanderbilt, Phys. Rev. B 65, 035109 (2001). The Wannier functions are generated by the code developed by A. A. Mostofi, J. R. Yates, N. Marzari, I. Souza and D. Vanderbilt, (http://www.wannier.org/) for the energy window -2.2 $\mathrm{eV}<\epsilon_{k}-E_{F}<3.6 \mathrm{eV}$, where $\epsilon_{k}$ is the eigenenergy of the Bloch states and $E_{F}$ the Fermi energy.

27 K. Momma and F. Izumi, J. Appl. Crystallogr. 41, 653 (2008).

${ }^{28}$ K. Kuroki, S. Onari, R. Arita, H. Usui, Y. Tanaka, H. Kontani, and H. Aoki, Phys. Rev. Lett. 101, 087004 (2008).

29 A five orbital model for $\mathrm{BaFe}_{2} \mathrm{As}_{2}$ was constructed in ref 34, which has a different orbital character in the $\alpha_{1}$ Fermi surface compared to our model.

30 T. Miyake , K. Nakamura, R. Arita, and M. Imada, J. Phys. Soc. Jpn. 79, 044705 (2010).

31 M. Rotter, M. Tegel, D. Johrendt, Phys. Rev. Lett. 101, 107006 (2008).

32 K. Suzuki, H. Usui, K. Kuroki , J. Phys. Soc. Jpn. 80, $013710(2010)$

33 M. Rotter, M. Tegel, D. Johrendt, I. Schellenberg, W. Hermes and R. Pöttgen, Phys. Rev. B 78, 020503 (2008).

34 S. Graser, A. F. Kemper, T. A. Maier, H.-P. Cheng, P. J. Hirschfeld, and D. J. Scalapino, Phys. Rev. B 81, 214503 (2010).

35 J. T. Park, D. S. Inosov,A. Yaresko, S. Graser, D. L. Sun, Ph. Bourges, Y. Sidis, Y. Li, J.-H. Kim, D. Haug, A. Ivanov, K. Hradil, A. Schneidewind, P. Link, E. Faulhaber, I. Glavatskyy, C. T. Lin, B. Keimer, and V. Hinkov, Phys. Rev. B. 82, 134503 (2010).

36 J. Knolle, I. Eremin, and R. Moessner Phys. Rev. B 83, 224503 (2011)

37 A. N. Yaresko, G.-Q. Liu, V. N. Antonov, and O. K. Andersen, Phys. Rev. B 79, 144421 (2009).

${ }^{38}$ K. Suzuki, H. Usui, and K. Kuroki, to be published in the proceedings of LT26

39 T. Dulguun, H. Mukuda, H. Kinouchi, M. Yashima, Y. Kitaoka, T. Kobayashi, S. Miyasaka, and S. Tajima, arXiv: 1108.4480 . 
${ }^{40}$ Y. Zhang, Z. R. Ye, Q. Q. Ge, F. Chen, J. Jiang, M. Xu, B. P. Xie, and D. L. Feng, arXiv:1109.0229.

${ }^{41}$ H. Kawano-Furukawa, C. J. Bowell, J. S. White, R.W. Heslop, A.S. Cameron, E.M. Forgan, K. Kihou, C. H. Lee,
A. Iyo, H. Eisaki, T. Saito, H. Fukazawa, Y. Kohori, R. Cubitt, C. D. Dewhurst, J. L. Gavilano and M. Zolliker, Phys. Rev. B 84, 024507 (2011). 